\title{
Effects of Personal Health Record on Patient Healthcare Outcomes
}

\author{
Bonita R Payne ${ }^{1}$ and Sajeesh Kumar ${ }^{2 *}$ \\ ${ }^{1}$ MHIIM, Research Scholar, Institute for Health Outcomes and Policy Research, University of Tennessee Health Science Center, USA \\ ${ }^{2} \mathrm{PhD}$, Associate Professor, Department of Health Informatics \& Information Management, University of Tennessee Health Science Center, \\ USA
}

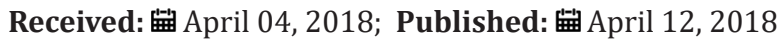

*Corresponding author: Sajeesh Kumar PhD, Associate Professor, Department of Health Informatics \& Information Management, University of Tennessee Health Science Center,920 Madison Avenue Suite 518, Memphis, Tennessee 38163, USA,

Email: skumar10@uthsc.edu

\begin{abstract}
Electronic transmission of data is on the rise, due to the Affordable Care Act and provisions to make healthcare information more accessible, complete, and transparent. The Personal Health Record (PHR) application is a tool used to provide assistance to the goal of patient-centered and patient-centric care. Its purpose is to encourage consumers to become more participatory and informed in their healthcare treatment and healthcare needs. Since its inception, it has been questionable as to whether consumers and providers are seeing any improvements in the services provided or the care rendered. Since, the development and implementation of PHR applications, there has been some resistance from consumers for concerns of privacy and security of their information. This paper will discuss the uses of the PHR among consumers and providers and whether its use has impacted accessibility, services, and overall healthcare treatment/outcomes.
\end{abstract}

Keywords: Patient Health Portals; Electronic Health Record; Effectiveness

Abbreviations: PHR: Personal Health Record, ACA: Affordable Care Act, EHR: Electronic Health Records, PHR: Positive Healthcare Outcomes. NSBE: National Society of Black Engineers

\section{Introduction}

In this era, electronic transmission/communications delivers instantaneous correspondence worldwide. In fact, in 2011, The Affordable CareAct(ACA) signed intolaw by PresidentBarack Obama, legitimatized this concept into the healthcare industry. Within the passing of this law, the mandate to develop Electronic Health Records (EHR) was implemented. One objective of this enactment is to provide patient-centered care. As a result, development of additional technologies (ie. applications) was created to allow patient/consumer-centered care. The Personal Health Record (PHR) application is a tool used to provide assistance to the goal of patient-centered/centric care. Its purpose is to encourage consumers to become more participatory and informed in their healthcare treatment and healthcare needs. As, a new technology, the PHR can increase positive healthcare outcomes for patients, physicians, and the healthcare industry as a whole. Currently, there has been low participation and advertisement of the PHR, due to patients/consumers concerns: data security, accuracy of the clinical information stored in the PHR, and challenges with keeping information updated Cocoslia [1]. The PHR is a tool designed with the objective to encourage consumers to share the responsibility of managing and participating in their healthcare treatment. The PHR as defined by the Markle Foundation: An electronic application through which individuals can access, manage and share their health information, and that of other for whom they are authorized, in a private, secure and confidential environment Tang et al.[2].

\section{Background}

Since, the development and implementation of PHR applications, there has been some resistance from consumers for concerns of privacy and security of their information. Since its inception, it has been questionable whether consumers and providers are seeing any improvements in the treatment or services provided. Although, 
the PHR is a relatively new tool in the healthcare industry; it can provide consumers with some timesaving benefits such as, access to a wide variety of credible health information, data, and knowledge Tang [2]; however, there has been minimal use. The quality of healthcare services has been a key component in providing patient satisfaction. It has been named as the focal point in decreasing healthcare cost in the United States. Patient-centered care is a core component of the Institute of Medicine's (IOM) quality aim and the Affordable Care Act (ACA) of 2011 Agarwal et al. [3]. Determining whether maintaining a PHR promotes healthier patient outcomes is critical to patient-centered healthcare. By establishing uses of this technology, healthcare providers can educate consumers of its benefits, as well as, increase patient communication, providerpatient relationships, patient compliance, and empower the patient to become an active player in their own healthcare treatment. The intent of this study is to determine whether patient participation and accessibility to healthcare information has effectively or ineffectively resulted in healthier patient outcomes.

The research question is:

a) Does Maintaining a PHR promote healthier patient outcomes?

The analysis of the survey will focus on the familiarity of PHRs, it uses, and impact on patient care.

\section{Methodology}

Research for this proposal was conducted via the University of Tennessee Health Science Center's library e-databases: CINHAL AND PubMed. PubMed offered a variety of full-text articles from the basic definition of the PHR to a variation of chronic illnesses (ie. Diabetes, CHF, etc.). It was found that CINHAL had fewer articles pertaining to the PHR health benefits or outcomes. The search for articles with the key words PHR or Personal Health Records was conducted and yielded 11 articles. Of those 11 articles, five of the articles contained information relative to the topic. The six articles that were excluded contained subject matters with same acronyms but different subject (ie. Protected Health Record) and were none supportive of my topic. After approval of the survey tool, selection of a database was initiated. Upon review of the available databases, it was found that Survey Monkey, an online cloud-based tool used for developing and conducting surveys, would meet the needs of this study. A data collection instrument was developed in Survey Monkey incorporating the variables outlined in the research design section. The survey was administered through Survey Monkey utilizing a Web link that provided access to the survey tool. This data collection method was found to straightforward and efficient. The subject sample selected was of convenience and selected to give a diverse distribution to the hypothesis and reliability of the study. Participants were randomly selected based merely on their willingness to participate in the survey. Population and sample chosen is due to time constraints and the necessity to develop a deeper understanding of consumer uses.

\section{Data Collection Procedures}

A questionnaire of 10 questions relating to the uses of PHRs was dispersed and/or administered via face-to-face interview and online Web-link via Survey Monkey. Survey data will be analyzed by tabulating response rates, frequencies of variables, and statistical testing. For the face-to-face survey participants, a consent form along with hard copies of questionnaire were administered to members of the National Society of Black Engineers (NSBE) that indicated an interest in participating in the survey on October 14, 2015. Seven questionnaires were distributed and completed. On October 25, 2015, a link to the survey with cover statement attached was dispersed to 347 e-mail addresses. Forty-three surveys were completed by the deadline of November 1, 2015. After the deadline, Survey Monkey's data export tool was used to export the aggregated data to Microsoft Excel 2010 for quantitative analysis of data captured. From the data, frequency tables and graphs were created to give visual analysis of survey variables.

\section{Result}

\section{Response Rate of Population}

A total of seven face-to-face questionnaires were distributed and completed for a response rate of $2 \%$. The first request for survey participation via Survey Monkey Web-link yielded 29 responses with a response rate of $8.4 \%$. The second survey participant request via Survey Monkey yielded 14 responses with a response rate of $4 \%$. Total overall online survey responses were 43 responses, totaling $12.4 \%$ response rate. Combined total faceto-face and online survey were 50 responses yielding a response rate of $14.4 \%$.

\section{Analysis and Discussion}

The response to the survey indicates that $24 \%$ of the population had some working knowledge of the PHR, $16 \%$ were familiar with it, $34 \%$ were somewhat familiar, and $20 \%$ had never heard of it. When asked how many health care providers the have $54 \%$ reported have at least 1-2 healthcare providers and one participant with 9 or more healthcare providers. Sixty-eight percent of the participants' report traveling out of the city, state, or country 1 to 3 times a year. When it came to the question \#8 of the survey, $36 \%$ of the respondents stated that they would not be able to produce this information in a timely manner in the event of an emergency; $12 \%$ immediately, $22 \%$ likely, $14 \%$ likely, and $8 \%$ somewhat likely. Lastly, $26 \%$ of the respondents spend 1-2 hours collecting medical information. Six percent 3--5 hours, 4\% 1-2 days, and 64\% none at all.

\section{Limitations}

Initially, the limitations of this study were the availability to historical research of the PHR. The search for PHR yielded predominately articles on the Protected Health Record. (PHR). Numerous PHR search results populated articles on the patient portal due to the lack of understanding of PHR versus patient portal, as well as, limited evaluations of PHR uses. The distribution 
of online web link limited survey responses due to opt-out of Survey Monkey website and deactivated email addresses.

\section{Conclusion and Recommendations}

The PHR is not a new tool for storing and maintaining healthcare information. It has been in existence since the early 1900s.The methods for health record keeping has evolved from the traditional alternative of binders and shoeboxes to electronic format. Evidence-based research has identified that those who traditionally maintained health documentation are those individuals with chronic illnesses. As a result, it decreased their health care costs tremendously by avoiding duplication of testing, and getting involved in the medical decision making process. Unfortunately, the natural disaster of Hurricane Katrina heightened the awareness of the necessity of EHRs and PHRs alike. The destruction of millions of medical record caused reduction in medical care, as well as, some deaths. With a PHR, the patient has control and total authorization of the management of healthcare documentation The PHR can be a life saver in medical emergencies; if needed request for medical can be managed by the patient versus waiting on service provider. This study has shown the benefits of accessibility when the consumer is in control of their own medical documentation. As technology continues to evolve, consumers will be pushed to keep up with advancements. Due to regulatory standards and laws that require accessibility to healthcare information, the providers, consumers, and employers will be forced to assist in making healthcare evolving trends a reality. PHRs is not a new thing; it has just evolved to electronic access. For those who are familiar with PHRs, historical and current research has provided evidence that having a PHR will benefit the consumer, the provider, employers, and cut the cost of healthcare for individuals and the healthcare industry. As a solution to some concerns, employers and healthcare payers are offering this technology to patients/employees to promote greater patient engagement in health and well-being Chrischilles et al. [4].

\section{Implications of the Study}

As, a new technology, the PHR can, in fact, promote positive healthcare outcomes for patients, physicians, and the healthcare industry as a whole. PHRs solicit active participation of the consumer to get involved in their healthcare decision-making and treatments, ultimately, leading to informed decisions and positive healthcare outcomes. The quality of healthcare services provided has been a key component in providing patient satisfaction and has been named as the focal point in decreasing healthcare cost in the United States. The initiative to use the PHR meets the requirements of federal and state mandates. The goal is to provide patient-centered care. To stimulate the use of PHRs, physicians and healthcare professionals need to educate patients/consumers on the uses of PHRs. By establishing uses of this technology, healthcare providers can educate consumers of its benefits, as well as, increase patient communication, provider-patient relationships, patient compliance, and empower the patient to become an active player in their own healthcare treatment. In turn healthcare providers and consumers will gain patient satisfaction and better healthcare outcomes. With more marketing and initiation from healthcare professionals, consumers will unite in the effort to promote patientcentered care, ultimately, leading to positive healthcare outcomes $[5,6]$.

\section{References}

1. Cocoslia M, Archer N (2014) Perceptions of chronically ill and healthy consumers about electronic personal health records: a comparative empirical investigation. BMJ Open 4(7): e005304.

2. Tang Paul C, Ash Joan S, Bates David W, Overhage J Marc (2006) Personal Health Records: Definitions, Benefits, and Strategies for Overcoming Barriers to Adoption. J Am Med Inform Assoc 13(2): 121-126.

3. Agarwal Ritu, Anderson Catherine, Zarate Jesus, Ward Claudine (2013) If We Offer it, Will They Accept? Factors Affecting Patient Use Intentions of Personal Health Records and Secure Messaging. Journal of Medicine Internet Research 15(2): e43.

4. Chrischilles Elizabeth A, Hourcade Juan Pablo, Doucette William, Eichmann David, Grylak Brian, Lorentzen Ryan, et al. (2014) Personal Health Records: a Randomized trial of effects on Elder Medication Safety. Journal of Medical Information Association 21(4): 679-686.

5. Roop Elizabeth S (2009, October 26) The PHR-Moving Consumers from Pawns to Kings. For The Record 21(20): 14.

6. Sands Daniel Z (2006) Personal Health Records: Definitions, Benefits, and Strategies for Overcoming Barriers to Adoption. Journal of the American Medical Informatics Association 13(2): 121-126.

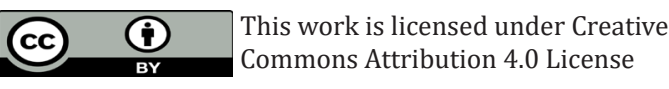

To Submit Your Article Click Here:

Submit Article

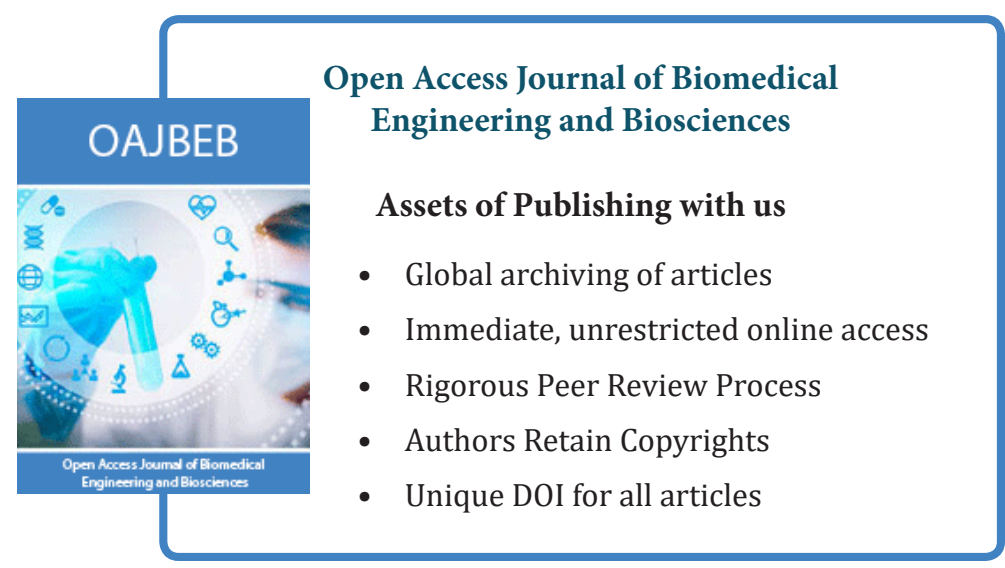

